\title{
Estimativa de Parâmetros Genéticos, Fenotípicos e Ambientes para as Produções de Leite no Dia do Controle e em 305 Dias de Lactação de Vacas da Raça Gir ${ }^{1}$

\author{
Ivan Luz Ledic ${ }^{2}$, Humberto Tonhati ${ }^{3}$, Rui da Silva Verneque ${ }^{4}$, Lenira El Faro ${ }^{5}$, Mário Luiz \\ Martinez ${ }^{4}$, Cláudio Nápolis Costa ${ }^{4}$, Jonas Carlos Campos Pereira ${ }^{6}$, Leonardo de Oliveira \\ Fernandes $^{7}$, Lúcia Galvão de Albuquerque ${ }^{3}$
}

\begin{abstract}
RESUMO - Dados de 32.779 controles mensais, de 3.605 lactações em 305 dias (PL305), de 2.082 vacas Gir, filhas de 281 touros, com partos ocorridos de 1987 a 1999, em 11 rebanhos, foram usados com o objetivo de verificar a viabilidade de utilização da produção de leite no dia do controle (PLDC1 a PLDC10) em avaliações genéticas da raça Gir. Foram realizadas análises bivariadas entre as PLDC1 a PLDC10 e PL305, usando para estimar os componentes de (co)variâncias o método de máxima verossimilhança restrita, sob modelo animal, incluindo as três primeiras lactações como medidas repetidas de uma mesma vaca, diferenciadas conforme o grupo contemporâneo de rebanho-ano-estação, de acordo com a idade da vaca ao parto e o intervalo parto-primeiro controle na PLDC1. As médias observadas e os respectivos desvios-padrão $(\mathrm{kg})$ para PLDC1 a PLDC10 e PL305 foram: 11,97 $\pm 4,64 ; 11,93 \pm 4,68 ; 10,98 \pm 4,40 ; 10,18 \pm 4,12$; $9,66 \pm 3,88 ; 9,20 \pm 3,69 ; 8,63 \pm 3,51 ; 8,08 \pm 3,33 ; 7,59 \pm 3,27 ; 7,22 \pm 3,15$ e $2.746,17 \pm 1.299,90$. A duração de lactação foi de $273,72 \pm 48,95$ dias. As estimativas de herdabilidade variaram nas PLDC de 0,24 a 0,14, sendo maior no primeiro controle e decrescendo até o décimo. A herdabilidade da PL305 foi de 0,19. As estimativas de correlações genéticas entre as PLDC e PL305 variaram de 0,85 a 1,00. As correlações genéticas entre PLDC1 a PLDC9 com PLDC6 a PLDC10 foram acima de 0,80, à exceção entre PLDC9 com PLDC1 e PLDC10 com PLDC1, PLDC2, PLDC3 e PLDC6, que foram inferiores. As correlações fenotípicas tiveram valores intermediários entre as correlações genéticas e as residuais (menores). Considerando a eficiência relativa de seleção, esta mostrou que se baseada nas PLDC2 a PLDC4 ocorrerá a mesma ou melhor resposta que se praticada na PL305.
\end{abstract}

Palavras-chave: bovino de leite, controle leiteiro, eficiência de seleção, melhoramento genético

\section{Genetic, Phenotypic and Environmental Parameters for Milk Production at the Control Day and 305 Days of Lactation of Gir Breed Cows}

\begin{abstract}
Data from 32,779 monthly milk production, from 3,605 lactations until 305 days (PL305), from 2,082 Gir cows, sired by 281 bulls, calving from 1987 to 1999, in 11 herds, were used to verify the viability of using daily milk production control (PLDC) to perform genetic evaluations within Gir breed. Bivariate analysis on PLDC1 to PLDC10 and PL305 were performed utilizing the restricted maximum likelihood method within an animal model, which included the three first lactations as replicate measures of the same dams unit. Animals were grouped according to the criterion of contemporaneous group of herd-year-season, and according to calvingage and to the interval calving-first control in PLDC1. The observed averages $(\mathrm{kg})$ and the respective standard deviations for PLDC1 at PLDC10 and PL305 were: $11.97 \pm 4.64 ; 11.93 \pm 4.68 ; 10.98 \pm 4.40 ; 10.18 \pm 4.12 ; 9.66 \pm 3.88 ; 9.20 \pm 3.69 ; 8.63 \pm 3,51 ; 8.08 \pm 3.33 ; 7.59 \pm 3.27$; $7.22 \pm 3.15$ and $2746.17 \pm 1299.90$. The average lactation length was $273.72 \pm 48.95$ days. Heritability estimates for PLDC based on bivariate analysis ranged from 0.24 to 0.14 , decreasing from the first to the tenth control; the heritability estimate for PL305 was 0.19 . Estimates of the genetic correlations between PLDC and PL305 varied from 0.85 to 1.00. The estimates of the genetic correlations between PLDC1 to PLDC9 with PLDC6 to PLDC10 were greater than 0.80, except the correlations between PLDC9 with PLDC1, PLDC10 with PLDC1, PLDC2, PLDC3 and PLDC6. The estimates of the phenotypic correlations resulting in intermediate value between genetic and environmental correlations (smaller). The results of genetic trend indicate that indirect selection for PLDC2 to PLDC4 was more efficient than direct selection for PL305.
\end{abstract}

Key Words: animal breeding, dairy cattle, milking control, test day

\footnotetext{
1 Parte da tese de Doutorado do primeiro autor apresentada à FCAV/UNESP de Jaboticabal.

2 Pesquisador da EMBRAPA Gado de Leite, Caixa Postal 351, 38001-970, Uberaba, MG. E.mail: ledic@enetec.com.br

3 Professor da FCAV/UNESP, Via Prof. Paulo Castellane s/n, 14884-900, Jaboticabal, SP.

4 Pesquisadores da EMBRAPA Gado de Leite, R. Eugênio do Nascimento 610, 36038-330, Juiz de Fora, MG.

5 Pesquisadora do IZ, Av. Bandeirantes 2419, 14001-970, Ribeirão Preto, SP.

6 Professor da EV/UFMG, Av. Antônio Carlos 6627, 30123-970, Belo Horizonte, MG.

7 Pesquisador da EPAMIG, Caixa Postal 351, 38001-970, Uberaba, MG.
} 


\section{Introdução}

O Brasil, com 8.547.403 km2, ocupa, em extensão territorial, 20,8\% das Américas e 47,7\% da América do Sul (Almanaque Abril, 1999). As áreas de pastagem ocupam cerca de $76 \%$ da superfície usada pela agricultura, o que corresponde a $21 \%$ do território brasileiro, algo em torno de 177 milhões de hectares (Censo, 2000). Apesar desta 'geopotencialidade', os sistemas de produção em pasto utilizados nas condições brasileiras são tradicionalmente desprovidos de planejamento e controle, sendo menos eficientes, em relação à muitos países, em termos de produtividade e com custos de produção mais elevados (Faria \& Corsi, 1986).

O rebanho bovino brasileiro é constituído de 102 milhões de animais criados na pecuária de corte e 45 milhões (31\% do total) dedicadas à produção leiteira (Anualpec, 2000). O total de vacas em reprodução do gado leiteiro é de cerca de 14 milhões. É o primeiro rebanho leiteiro comercial do mundo $(6,1 \%$ do total de vacas), mas em conseqüência da baixa produtividade (19 bilhões de litros de leite/ano), ocupa modesta posição $(4,0 \%)$ com respeito ao total produzido. Segundo Viana (1999), o Brasil possui um dos maiores rebanhos do mundo, porém com média produtiva de leite de $3,8 \mathrm{~kg} /$ vaca/dia, abaixo da média mundial (7,8 kg/dia)e 3,6 vezes menor que a média dos países desenvolvidos (13,8kg/dia).

Uma das razões apontadas para este desempenho inferior é a utilização de animais e de sistemas de criação inadequados para a produção de leite. Gomes (1997) estimou que 54\% dos produtores de leite produzem até 50 litros/dia e respondem com somente $10 \%$ da produção. No outro extremo, produzindo mais de 200 litros/dia, estão apenas $10 \%$ do produtores, que, no entanto, participam com $50 \%$ da produção total de leite.

Não existe opinião consensual no meio técnico quanto ao tipo de sistema de produção e de gado mais conveniente às nossas condições de criação. Entretanto tem ocorrido aumento expressivo da produtividade do rebanho nacional, especialmente entre criadores de gado elite e mesmo de mestiços, com produções equiivalentes a dos países do primeiro mundo, fato este desconsiderado em virtude de se utilizar a média para expressar a produtividade de uma população tão dispersa. O Brasil possui populações bovinas leiteiras de alto valor genético e qualificadas para obtenção de indicadores de produtividade comparáveis aos obtidos nos países mais desenvolvidos (Pereira, 1998).

Sem considerar outros fatores, o aumento da produtividade passa necessariamente pela busca de animais de valor genético superior para produção de leite, adaptados às condições ambientes e sócioeconômicas do país. A raça Gir, selecionada para leite, dentre outras zebuínas leiteiras e seus mestiços, tem apresentado desempenhos satisfatórios nas nossas condições de criação (Ledic, 1985).

Cabe salientar que o Brasil possui apenas 26.000 animais da raça Gir, distribuídos em 397 rebanhos. Das cerca de 8.000 vacas em reprodução, 50\% pertencem a rebanhos que executam controle leiteiro oficial, em 57 propriedades rurais, com média de $3.321 \mathrm{~kg}$ de leite por lactação (Ledic, 2000).

Apesar disto, esta raça é muito importante na pecuária leiteira nacional, participando com 31,04\% da comercialização do sêmen nacional das raças leiteiras, segunda em volume de vendas (316.903 doses), de acordo com os dados da Asbia (2002). Foi, ainda, a primeira a ter teste de progênie de touros e avaliação genética de vacas implantado no Brasil (Ledic, 1996), tendo dez grupos de touros avaliados (84 animais) e seis grupos em avaliação (81 touros), segundo relataram Verneque et al. (2002). Além disto, a raça Gir no Brasil, conforme reportou Ledic (1995), tem rebanhos com controle leiteiro oficial desde 1964, sendo a segunda raça em volume de animais com registros de lactação (23\% do total), possuindo o Arquivo Zootécnico Nacional de Gado de Leite, para a raça Gir, mais de 36.000 lactações obtidas de cerca de 14.000 vacas (Ledic \& Tonhati, 1998).

A medida padrão convencionada de produção de leite é o rendimento em 305 dias de lactação (PL305) e a seleção de reprodutores, das raças leiteiras, tem sido baseada neste critério produtivo. A produção de leite no dia do controle (PLDC) tem sido, também, utilizada para estimar parâmetros genéticos e ambientes visando auxiliar a seleção em gado de leite. Os estudos sobre a viabilidade de utilizaçãoda PLDC para estimarparâmetros genéticos, fenotípicos e ambientes das características produtivas visando auxiliar a seleção em gado de leite são bem antigos (Madden et al., 1959; Searle, 1961; Lamb \& McGilliard, 1967; Keown \& Van Vleck, 1971).

A falta de recursos computacionais era fator limitante para estes estudos, pois após o cálculo da produção na lactação, os controles parciais mensais eram eliminados por falta de espaço computacional para armazenamento destas informações (Ptak \& Schaeffer, 1993). Além disto, o método estatístico mais indicado para estimar componentes de (co)variância de dados não balanceados das PLDC é o de máxima verossimilhança restrita (REML), o que

R. Bras. Zootec., v.31, n.5, p.1953-1963, 2002 
exige processadores mais velozes e maior capacidade de random access memory (RAM), para execução dos algorítmos e processamento dos dados (Ptak \& Schaeffer, 1993; Swalve, 1994; Rekaya et al., 1995; Firat et al., 1997a,b; Jamrozik et al., 1997; Vargas et al., 1998; Ferreira, 1999).

Os notáveis avanços ocorridos na área de informática possibilitaram o desenvolvimento de equipamentos com processadores mais velozes e otimizados, com maior capacidade dos dispositivos de armazenamento (Meyer, 1991; Verneque, 1994; Wiggans \& Goddard, 1997). Este fato viabilizou a retomada dos trabalhos que propunham a utilização da PLDC em estudos de seleção para gado de leite.

Vários modelos estatísticos têm sido propostos para analisar a PLDC. Além dos trabalhos já citados, destacam os de Van Vleck \& Henderson (1961a e 1961b); Danell (1982); Stanton et al. (1992); Gadini (1997); Machado (1997); Strabel \& Szwaczkowski (1997); Kettunen et al. (1998). Esses trabalhos mostraram existirem altas correlações entre os controles parciais e a produção total de leite em 305 dias de lactação, tornando exequível a utilização de menor número de controles por lactação como critério de seleção para características produtivas em gado de leite.

Estes modelos, utilizando a PLDC, poderão incluir além do efeito peculiar para todas as vacas sobre o mesmo dia de controle, efeitos de meio associados com uma particular PLDC como: intervalo parto-primeiro controle (Danell, 1982), prenhez (Trus \& Buttazzoni, 1990), período de serviço (Wiggans \& Goddard, 1997), dias de gestação (Van Tassel et al., 1992), ordem do parto (Stanton et al., 1992; Reents et al., 1995), intervalo de controles (Pander et al., 1992). Por outro lado, se vacas são agrupadas de acordo com o nível de produção, como forma de tratamento preferencial, então este agrupamento poderá ser incluído no modelo, se for conhecido (Ferreira, 1999). Na PL305, a maioria desses efeitos são assumidos circunstanciais e não têm como serem considerados no modelo e ficam no resíduo.

Castle \& Searle (1961) verificaram que os controles bimestrais têm a mesma utilidade para eliminar vacas do rebanho que controles mensais, sendo o custo $40 \%$ menor, podendo ser ainda mais reduzido caso se possa executar menos controles durante a lactação. Ainda hoje, entretanto, são necessárias novas pesquisas para que a PLDC possa ser recomendada e efetivamente empregada, principalmente porque faltam informações a respeito dos parâmetros genéticos de lactações parciais de rebanhos leiteiros nos trópicos (Ribas \& Perez, 1990).

No Brasil, embora a literatura apresente alguns estudos sobre outros aspectos da curva de lactação das raças leiteiras e modelos de ajustes, não se tem conhecimento sobre estudos com a PLDC, a não ser os trabalhos de Machado (1997) e Ferreira (1999), com registros de produção de leite de vacas da raça Holandês, controladas pela Associação Brasileira de Criadores e Associação dos Criadores de Gado Holandês de Minas Gerais, respectivamente. Com animais das raças zebuínas, exploradas para leite, não se encontrou qualquer referência na literatura. A falta de pesquisas, nesta área, decorre do pequeno número de animais sob controle leiteiro no Brasil (menos de $0,2 \%$ das vacas em lactação), devido ao alto custo para este procedimento e à falta de qualquer estímulo para sua implementação rotineira.

Assim, este estudo foi conduzido com o objetivo de verificar a possibilidade de se utilizarem as PLDC como critério de seleção em avaliações genéticas de animais da raça Gir, estimando parâmetros como herdabilidades e correlações genéticas, fenotípicas e ambientes das PLDC e PL305.

\section{Material e Métodos}

Os controles individuais de produção de leite de vacas Gir, utilizados neste trabalho, foram coletados mensalmente pela Associação Brasileira de Criadores e Associação Brasileira de Criadores de Zebu.

$\mathrm{O}$ arquivo base, do qual foram extraídas as informações utilizadas neste estudo, continha 55.717 registros de controle leiteiro mensal, obtidos em duas ordenhas diárias, de partos ocorridos de 1973 a 1999 , de diversas ordens de parição de 17 rebanhos e estavam armazenados no banco de dados do Arquivo Zootécnico Nacional, gerenciado pela Embrapa Gado de Leite. Este arquivo possuía igualmente a produção de leite em 305 dias de lactação (PL305) calculada de acordo com o método oficial reconhecido pelo Ministério da Agricultura (Brasil, 1986).

As características estudadas foram as produções de leite obtidas no dia do controle leiteiro (PLDC1 a PLDC10) e a produção de leite em 305 dias de lactação (PL305), das três primeiras lactações. Do arquivo base, foram gerados sub-arquivos de trabalho para verificações e consistências dos dados, utilizando para tal fim procedimentos disponíveis no

\section{R. Bras. Zootec., v.31, n.5, p.1953-1963, 2002}


software Sas (1990). Outras consistências e cálculos, bem como tabelas e gráficos, foram efetuadas utilizando os programas Word e Excel da Microsoft (1997).

Inicialmente, foram eliminadas informações de lactações com menos de três controles mensais, rebanhos e anos com menos de 100 lactações.

Posteriormente foram incluídas as variáveis idade da vaca ao parto, intervaloparto-primeirocontrole leiteiro(ipc) e estações do parto e do controle leiteiro (estação 1 - meses de janeiro, fevereiro e março; Estação 2 - meses de abril, maioe junho; Estação 3 - meses de julho, agostoe setembro; Estação 4 - meses de outubro, novembro e dezembro).

As estações (do parto ou do controle) foram agrupadas dentro de rebanho e do ano (do parto ou do controle), criando-se as sub-classes de grupos contemporâneos de rebanho-ano-estação do parto (raep305) e rebanho-ano-estação do controle (raec1 a raec10).

Foram, então, impostas novas restrições aos dados, eliminando registros de vacas com idade ao parto inferior a 24 meses e superior a 120 meses, produções do primeiro controle com intervalo parto-primeiro controle leiteiro inferiores a 5 dias e superiores a 35 dias, grupos contemporâneos com menos de 10 observações e touros com menos de duas filhas.

Foi gerado, finalmente, o arquivo para as análises, que contabilizou 32.779 controles mensais de 3.605 lactações de 2.082 vacas, filhas de 281 touros, com partos ocorridos de 1987 a 1999 em 11 rebanhos, contendo as seguintes variáveis: número da vaca, número do pai, número da mãe, ordem do parto, raec1 a raec10, raep305, idade da vaca ao parto, ipc, duração da lactação, PLDC1 a PLDC10 e PL305.

Foi constituído, para formação da matriz de numeradores dos coeficientes de parentesco (NRM), um arquivo de pedigrees, utilizado em todas as análises, contendo a identificação da vaca, do pai e da mãe, resultando em 2.082 pedigrees decifrados, com 3.407 animais diferentes e 9 animais que apresentaram endogamia média de 0,26.

Os componentes de variância, covariância e os parâmetros genéticos foram estimados pelo método de máxima verossimilhança restrita, sob modelo animal, utilizando o aplicativo MTDFREML (Multiple Trait Derivative-Free Restricted Maximum Likelihood), descrito por Boldman et al. (1995).

Nas análises bivariadas entre as PLDC e entre as PLDC e PL305, o modelo adotado foi o seguinte:

$$
\begin{gathered}
Y_{i j k l}=\mu+R_{A E}+\left[b_{1}\left(I_{i j}-I\right)+b_{2}\left(I_{i j}-I\right)^{2}\right]+ \\
{\left[c_{1}\left(D_{i j k}-D\right)+c_{2}\left(D_{i j k}-D\right)^{2}\right]+a_{i j k l}+a p_{i j k l}+e_{i j k l}}
\end{gathered}
$$

em que: $\mathrm{Y}_{\mathrm{ijkl}}=$ produção de leite para as características PLDC1 a PLDC10 e PL305; $\mu$ = média geral das características; $\mathrm{RAE}_{\mathrm{i}}=$ efeito fixo da i-ésima subclasse de rebanho-ano-estação do controle (PLDC1 a PLDC10) ou rebanho-ano-estação do parto (PL305); $b_{1}$ e $b_{2}=$ coeficientes de regressão linear e quadrático da produção $\mathrm{Y}_{\mathrm{ijkl}}$, em função da idade da vaca ao parto; $\mathrm{I}_{\mathrm{ij}}=$ efeito da $\mathrm{j}$-ésima idade da vaca ao parto; I = média da idade da vaca ao parto; $c_{1}$ e $c_{2}=$ coeficiente de regressão linear e quadrático da PLDC1, em função do intervalo parto-primeiro controle; $\mathrm{D}_{\mathrm{ijk}}=$ efeito do k-ésimo intervalo partoprimeiro controle (na $P L D C 1$ ); $\mathrm{D}=$ média do intervalo parto-primeiro controle (na $P L D C 1) ; \mathrm{a}_{\mathrm{ijkl}}=$ efeito aleatório da l-ésima vaca, $\sim\left(0, \sigma^{2}{ }_{\mathrm{a}}\right) ; \mathrm{ap}_{\mathrm{ijkl}}=$ efeito aleatório de ambiente permanente sobre a l-ésima vaca, $\sim\left(0, \sigma^{2}{ }_{\text {ap }}\right) ; \mathrm{e}_{\mathrm{ijkl}}=$ efeito aleatório residual associado à observação da vaca 1 , intervalo partoprimeiro controle $\mathrm{k}$ (na PLDC1), idade ao parto $\mathrm{j}$, rebanho-ano-estação i, $\sim\left(0, \sigma_{\mathrm{e}}^{2}\right)$.

Em termos matriciais, este modelo pode ser descrito como:

$$
\mathrm{Y}=\mathrm{X} \beta+\mathrm{Za}+\mathrm{Wap}+\mathrm{e}
$$

A notação matricial das análises com duas características é descrita da seguinte forma:

$$
\left[\begin{array}{l}
\mathrm{Y}_{1} \\
\mathrm{Y}_{2}
\end{array}\right]=\left[\begin{array}{ll}
\mathrm{X}_{1} & 0 \\
0 & \mathrm{X}_{2}
\end{array}\right]\left[\begin{array}{l}
\beta_{1} \\
\beta_{2}
\end{array}\right]+\left[\begin{array}{ll}
\mathrm{Z}_{1} & 0 \\
0 & \mathrm{Z}_{2}
\end{array}\right]\left[\begin{array}{l}
\mathrm{a}_{1} \\
\mathrm{a}_{2}
\end{array}\right]+\left[\begin{array}{cc}
\mathrm{W}_{1} & 0 \\
0 & \mathrm{~W}_{2}
\end{array}\right]\left[\begin{array}{l}
\mathrm{ap}_{1} \\
\mathrm{ap}_{2}
\end{array}\right]+\left[\begin{array}{l}
\mathrm{e}_{1} \\
\mathrm{e}_{2}
\end{array}\right]
$$

em que: $Y_{i}=$ vetor $n_{i} \times 1$, de $n_{i}$ observações de produções de leite da característica $\mathrm{i}, \mathrm{i}=$ PLDC1 a PLDC10 e PL305; $X_{i}=$ matriz $n_{i} \times p$, de incidência de $p$ níveis dos efeitos fixos de produções de leite da característica $i$; $\beta_{\mathrm{i}}=$ vetor $\mathrm{p}$ x 1 , de efeitos fixos da característica $\mathrm{i} ; \mathrm{Z}_{\mathrm{i}}=$ matriz bloco diagonal $n_{i} \times n_{i}$, de incidência dos valores genéticos, contendo "1" na característica i e animal $\mathrm{j}$; $a_{i}=$ vetor $n_{i} \times 1$, dos valores aleatórios genéticos dos animais da característica $\mathrm{i} ; \mathrm{W}_{\mathrm{i}}=$ matriz $\mathrm{n}_{\mathrm{i}} \times \mathrm{q}$, de incidência de q níveis dos efeitos aleatórios de ambiente permanente da característica $\mathrm{i} ; \mathrm{ap}_{\mathrm{i}}=$ vetor $\mathrm{q} \times 1$, dos efeitos aleatórios de ambiente permanente da característica i, não relacionados com os efeitos de $\mathrm{a} ; \mathrm{e}_{\mathrm{i}}=$ vetor dos efeitos aleatórios residuais da mesma dimensão de Y.

As pressuposições usuais para este modelo são:

$$
\operatorname{Var}\left[\begin{array}{l}
\mathrm{a} \\
\mathrm{ap} \\
\mathrm{e}
\end{array}\right]=\left[\begin{array}{lll}
\mathrm{G} & 0 & 0 \\
0 & \mathrm{P} & 0 \\
0 & 0 & \mathrm{R}
\end{array}\right]=\left[\begin{array}{ccc}
\mathrm{G}_{0} \otimes \mathrm{A} & 0 & 0 \\
0 & \mathrm{P}_{0} \otimes \mathrm{Im} & 0 \\
0 & 0 & \mathrm{R}_{0} \otimes \mathrm{I}_{\mathrm{n}}
\end{array}\right]
$$

\section{R. Bras. Zootec., v.31, n.5, p.1953-1963, 2002}


em que:

$\mathrm{G} 0=\left[\begin{array}{cc}\sigma_{\mathrm{a}}^{2} & \sigma_{\mathrm{aa}^{\prime}} \\ \sigma_{\mathrm{aa}} & \sigma_{\mathrm{a}^{\prime}}^{2}\end{array}\right]=$ matriz de (co)variância genética aditiva;

$\mathrm{P} 0=\left[\begin{array}{cc}\sigma_{\text {ap }}^{2} & \sigma_{\text {apap' }} \\ \sigma_{\text {apap' }} & \sigma_{\text {ap' }}^{2}\end{array}\right]=$ matriz de (co)variância dos efeitos ambientes permanentes;

$\mathrm{R} 0=\left[\begin{array}{ll}\sigma_{\mathrm{e}}^{2} & \sigma_{\mathrm{ee^{ \prime }}} \\ \sigma_{\mathrm{ee}} & \sigma_{\mathrm{e}^{\prime}}^{2}\end{array}\right]=$ matriz de (co)variância dos efeitos residuais.

$\mathrm{A}=$ matriz do numerador dos coeficientes de parentesco entre indivíduos; I = matriz identidade de ordem m (número de vacas) ou n (número de dados); $\otimes=$ operador do produto direto; $\sigma^{2}{ }_{\mathrm{a}}=$ variância genética aditiva das características; $\sigma^{2}{ }^{\mathrm{a}}$ ap $=$ variância de ambiente permanente das características; $\sigma_{\mathrm{e}}^{2}=$ variância residual das características; $\sigma_{a a^{\prime}}=$ covariância genética entre as características; $\sigma_{\text {ee' }}=$ covariância residual entre as características; $\sigma_{\text {apap' }}=$ covariância de ambiente permanente entre as características.

O BLUP de a e o BLUE das funções estimáveis de $\beta$ foram obtidos pela solução do sistema de equações do modelo misto (EMM) abaixo:

$\left[\begin{array}{ccc}X^{\prime} R^{-1} X & X^{\prime} R^{-1} Z & X^{\prime} R^{-1} W \\ Z^{\prime} R^{-1} X & Z^{\prime} R^{-1} Z+G^{-1} & Z^{\prime} R^{-1} W \\ W^{\prime} R^{-1} X & W^{\prime} R^{-1} Z & W^{\prime} R^{-1} W+P^{-1}\end{array}\right]\left[\begin{array}{l}\beta \\ a \\ a p\end{array}\right]=\left[\begin{array}{l}X^{\prime} R^{-1} y \\ Z^{\prime} R^{-1} y \\ W^{\prime} R^{-1} y\end{array}\right]$

As estimativas de herdabilidade e das correlações foram calculadas como:

herdabilidade $=\hat{\mathrm{h}}^{2}=\left(\frac{\hat{\sigma}_{\mathrm{a}}^{2}}{\hat{\sigma}_{\mathrm{f}}^{2}}\right)$;

correlação fenotípica $=\hat{\mathrm{r}}_{\mathrm{f}}=\frac{\hat{\sigma}_{\mathrm{ff}}}{\sqrt{\hat{\sigma}_{\mathrm{f}}^{2} \hat{\sigma}_{\mathrm{f}^{\prime}}^{2}}} ;$ correlação genética $=\hat{\mathrm{r}}_{\mathrm{a}}=\frac{\hat{\sigma}_{\mathrm{aa}}}{\sqrt{\hat{\sigma}_{\mathrm{a}}^{2} \sigma_{\mathrm{a}^{\prime}}^{2}}} ;$

correlação ambiente permanente $=\hat{\mathrm{r}}_{\mathrm{ap}} \frac{\hat{\sigma}_{\text {apap' }}}{\sqrt{\hat{\sigma}_{\text {ap }}^{2} \hat{\sigma}_{\text {ap' }}^{2}}} ;$

correlação residual $=\hat{\mathrm{r}}_{\mathrm{e}} \frac{\hat{\sigma}_{\mathrm{ee}}}{\sqrt{\hat{\sigma}_{\mathrm{e}}^{2} \hat{\sigma}_{\mathrm{e}^{\prime}}^{2}}}$

em que: $\hat{\sigma}_{\mathrm{f}}^{2}=\hat{\sigma}_{\mathrm{a}}^{2}+\hat{\sigma}_{\mathrm{ap}}^{2}+\hat{\sigma}_{\mathrm{e}}^{2} ; \hat{\sigma}_{\mathrm{ff}}=\hat{\sigma}_{\mathrm{aa}}+\hat{\sigma}_{\mathrm{apap}}+\hat{\sigma}_{\mathrm{ee}}$

A eficiência relativa de seleção (ERS) foi calculada segundo Ribas \& Perez (1990):

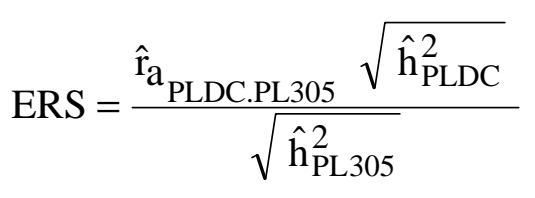

\section{Resultados e Discussão}

As médias observadas, os desvios-padrão e os coeficientes de variação para produção de leite no dia do controle leiteiro e para produção de leite em 305 dias de lactação, podem ser visualizadas na Tabela 1.

Os coeficientes de variação $(\mathrm{CV})$ foram elevados e aumentaram com o avançar da lactação, com valor mais elevado na PL305. Seriam, conforme Sampaio (1998), características muito instáveis.

Os CV foram maiores que os citados nos trabalhos de Albuquerque (1984), Gadini (1985, 1997), Machado (1997) e Ferreira (1999), os quais apresentaram valores de 20 a $30 \%$. Os altos CV encontrados refletem, principalmente, desuniformidade de produções entre os rebanhos estudados. A diferença entre as produções dos rebanhos chegou a $66 \%$ (de 5,80 a $16,84 \mathrm{~kg}$ ), 64\% (de 6,21 a 17,06 kg), 61\% (de 6,14 a $15,77 \mathrm{~kg}$ ), $59 \%$ (de $6,05 \mathrm{a} 14,78 \mathrm{~kg}$ ), $58 \%$ (de $5,85 \mathrm{a}$ $13,96 \mathrm{~kg}$ ), $56 \%$ (de 5,76 a $13,19 \mathrm{~kg}$ ), 55\% (de 5,62 a $12,41 \mathrm{~kg}$ ), 53\% (de 5,39 a $11,52 \mathrm{~kg}$ ), 51\% (de 5,23 a $10,56 \mathrm{~kg}$ ) e $52 \%$ (de 4,75 a $9,84 \mathrm{~kg}$ ) nas PLDC1 a PLDC10, respectivamente, e de 63\% na PL305 (de $1.615,62$ a $4.373,80 \mathrm{~kg}$ ). 
Tabela 1 - Número de observações, médias observadas, desvios-padrão e coeficientes de variação (CV) para produção de leite no dia do controle (PLDC1 a PLDC10) e para produção de leite em 305 dias de lactação (PL305) de vacas da raça Gir

Table 1 - Number of observations, observed averages, standard deviations and coefficients of variation (CV) for milk production at the control day (PLDC1 to PLDC10) and for milk production until 305 days (PL305) to Gir breed cows

\begin{tabular}{lcccc}
\hline $\begin{array}{l}\text { Característica } \\
\text { Trait }\end{array}$ & $\begin{array}{c}\text { Observações } \\
\text { Observations }\end{array}$ & $\begin{array}{c}\text { Média }(\mathrm{kg}) \\
\text { Average }(\mathrm{kg})\end{array}$ & $\begin{array}{c}\text { Desvio-padrão }(\mathrm{kg}) \\
\text { Standard deviation }(\mathrm{kg})\end{array}$ & $\mathrm{CV}(\%)$ \\
\hline PLDC1 & 2.719 & 11,97 & 4,64 & 38,77 \\
PLDC2 & 3.291 & 11,93 & 4,68 & 39,23 \\
PLDC3 & 3.256 & 10,98 & 4,40 & 40,07 \\
PLDC4 & 3.268 & 10,18 & 4,12 & 40,47 \\
PLDC5 & 3.160 & 9,66 & 3,88 & 40,16 \\
PLDC6 & 3.115 & 9,20 & 3,69 & 40,67 \\
PLDC7 & 3.019 & 8,63 & 3,51 & 41,21 \\
PLDC8 & 2.883 & 8,08 & 3,33 & 43,08 \\
PLDC9 & 2.631 & 7,59 & 3,27 & 43,63 \\
PLDC10 & 2.252 & 7,22 & 3,15 & 47,33 \\
PL305 & 3.185 & $2.746,17$ & $1.299,90$ & \\
\hline
\end{tabular}

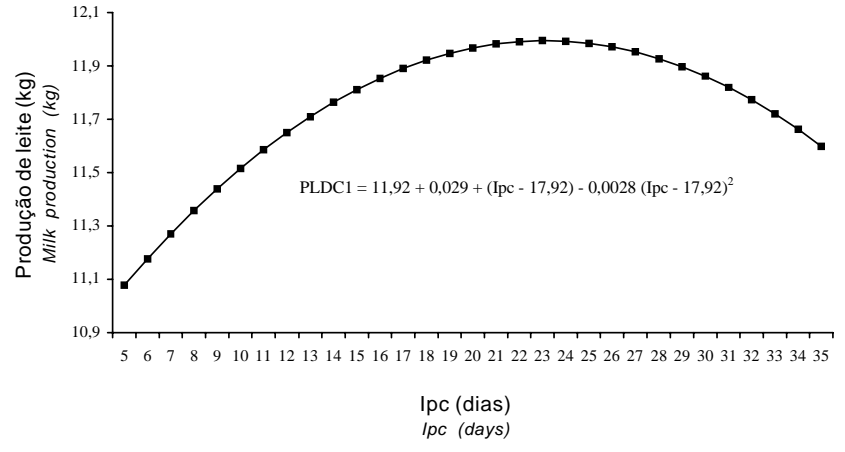

Figura 1 - Médias esperadas para a produção de leite no primeiro controle (PLDC1), em função do intervalo parto-primeiro controle (Ipc).

Figure 1 - Expected means for milk production in the first control (PLDC1), according to the interval calving first control (IPC).

Pode-se verificar, outrossim, que houve alteração conforme a fase da lactação, estando o pico de produção no primeiro controle. Observando o efeito do intervalo parto-primeiro controle (Ipc) sobre a PLDC1 (Figura 1), constata-se que a produção máxima ocorreu no $23^{\circ}$ dia pós-parto.

A redução, a partir do segundo controle, foi mais abrupta no terceiro e quarto controles (redução de 0,95 e $0,80 \mathrm{~kg}$ de leite em cada controle, respectivamente), mantendo declínio quase que constante a partirdaí (queda de aproximadamente $0,50 \mathrm{~kg}$ de leite por controle).

Cabe salientar que a PLDC10 foi elevada para considerar encerramento da lactação, principalmente em se tratando de animais zebuínos, representando queda de apenas 39,68\% em relação à PLDC1, estando muito acima da produção média do rebanho nacional.

A curva de lactação, observada neste estudo, não segue a mesma tendência observada por Schutz et al. (1990), Stanton et al. (1992), Machado (1997) e Ferreira (1999) para a raça Holandês, onde o pico de produção ocorreu entre o segundo e terceiro controles.

Confirmando nossos resultados, Rao \& Sundaresan, (1979), com rebanho Sahiwal e Bianchini Sobrinho (1984) e Gadini (1985) em rebanhos Gir, encontraram pico da produção no primeiro mês pósparto. Bianchini Sobrinho (1988) verificou que o pico da produção de leite na lactação de vacas Gir ocorreu antes do décimo dia, em um rebanho com controle leiteiro diário. Esta pode ser uma singularidade da raça Gir, um Bos taurus indicus, que atinge o máximo de produção leiteira já no primeiro mês da lactação.

As estimativas dos componentes de variância e covariância obtidas nas análises entre as PLDC e a PL305 estão expostas na Tabela 2.

Os resultados das estimativas de herdabilidade e das correlações obtidos entre as PLDC com a PL305 estão apresentados na Tabela 3.

Os menores valores da herdabilidade, a partir do primeiro controle, estão relacionados à maior redução da variância genética aditiva (diminuição de até $55 \%$; de $2,11 \mathrm{~kg}^{2}$ para $0,95 \mathrm{~kg}^{2}$ ) em relação à variância fenotípica (queda de até $29 \%$; de $9,28 \mathrm{~kg}^{2}$ para $6,56 \mathrm{~kg}^{2}$ ). Ocorreu também redução nas variâncias residual e de ambiente permanente com o decorrer da lactação (declínio de 32 e 16\%, respectivamente). 
Tabela 2 - Estimativas dos componentes de variância $\left(\mathrm{em}^{2} \mathrm{~kg}^{2}\right)$ genética $\left(\sigma_{\mathrm{a}}^{2}\right)$, ambiente permanente $\left(\sigma_{\mathrm{ap}}{ }^{2}\right)$, residual $\left(\sigma_{e}^{2}\right)$, fenotípica $\left(\sigma_{f}^{2}\right)$ e de covariâncias $\left(e m ~^{2}\right)$ genética $\left(\sigma_{a a^{\prime}}\right)$, ambiente permanente $\left(\sigma_{\text {apap }}\right)$, residual $\left(\sigma_{e e}\right)$ e fenotípica $\left(\sigma_{\mathrm{ff}}\right)$, das produções de leite no dia do controle (PLDC1 a PLDC10) e da produção de leite em 305 dias de lactação (PL305)

Table 2 - Estimated components of variance (in $\left.\mathrm{kg}^{2}\right)$ genetic $\left(\sigma_{a}^{2}\right)$, environmental permanent $\left(\sigma_{a p}^{2}\right)$, residual $\left(\sigma_{e}^{2}\right)$, phenotypic $\left(\sigma_{f}^{2}\right)$ and of covariance (in $\left.\mathrm{kg}^{2}\right)$ genetic $\left(\sigma_{a a^{\prime}}\right)$, environmental permanent $\left(\sigma_{\text {apap }}\right)$, residual $\left(\sigma_{e e^{\prime}}\right)$, phenotypic $\left(\sigma_{f f}\right)$ for milk production at the control day (PLDC1 to PLDC10) and for milk production until 305 days (PL305)

\begin{tabular}{lcccccccc}
\hline $\begin{array}{l}\text { Característica } \\
\text { Trait }\end{array}$ & $\sigma_{\mathrm{a}}{ }^{2}$ & $\sigma_{\mathrm{ap}}{ }^{2}$ & $\sigma_{\mathrm{e}}{ }^{2}$ & $\sigma_{\mathrm{f}}^{2}$ & $\sigma_{\mathrm{aa}}$ & $\sigma_{\text {apap' }}$ & $\sigma_{\text {ee' }}$ & $\sigma_{\mathrm{ff}{ }^{\prime}}$ \\
\hline PLDC1 & 1,99 & 1,37 & 5,06 & 8,42 & 465,04 & 538,82 & 607,67 & 1611,53 \\
PLDC2 & 2,11 & 1,99 & 5,18 & 9,28 & 525,74 & 635,79 & 722,63 & 1884,16 \\
PLDC3 & 1,90 & 1,94 & 5,06 & 8,90 & 509,05 & 665,79 & 758,96 & 1933,33 \\
PLDC4 & 1,75 & 1,72 & 4,39 & 7,87 & 494,18 & 651,28 & 793,17 & 1938,62 \\
PLDC5 & 1,41 & 2,22 & 3,93 & 7,56 & 444,38 & 723,86 & 796,95 & 1965,20 \\
PLDC6 & 1,37 & 1,80 & 4,02 & 7,20 & 425,04 & 674,99 & 764,40 & 1864,53 \\
PLDC7 & 1,29 & 1,97 & 3,60 & 6,86 & 426,66 & 699,85 & 731,75 & 1858,27 \\
PLDC8 & 1,17 & 2,06 & 3,51 & 6,74 & 430,35 & 698,57 & 710,31 & 1839,23 \\
PLDC9 & 1,20 & 1,68 & 4,20 & 7,08 & 412,74 & 644,80 & 776,05 & 1833,60 \\
PLDC10 & 0,95 & 1,92 & 3,69 & 6,56 & 319,82 & 665,32 & 618,30 & 1603,40 \\
PL305* & $150.976,37$ & $257.446,18$ & $356.245,03$ & $764.667,45$ & - & - & - & - \\
\hline
\end{tabular}

* Média obtida das 10 análises bivariadas.

*Average obtained from 10 bivariates analysis.

Tabela 3 - Estimativas das herdabilidades $\left(h^{2}\right)$ e das correlações genética $\left(r_{a}\right)$, ambiente permanente $\left(r_{\text {ap }}\right)$, residual $\left(r_{e}\right)$ e fenotípica $\left(r_{f}\right)$ entre produções de leite no dia do controle (PLDC1 a PLDC10) e a produção de leite em 305 dias de lactação (PL305) e as eficiências relativa de seleção (ERS)

Table 3 - Heritability $\left(h^{2}\right)$ and genetic $\left(r_{a}\right)$, environmental permanent $\left(r_{a p}\right)$, residual $\left(r_{e}\right)$ and phenotypic $\left(r_{f}\right)$ correlations estimated between milk production at the control day (PLDC1 to PLDC10) and milk production until 305 days (PL305) and selection relative efficiences (ERS)

\begin{tabular}{lcccccc}
\hline $\begin{array}{l}\text { Característica } \\
\text { Trait }\end{array}$ & $\mathrm{h}^{2}$ & $\mathrm{r}_{\mathrm{a}}$ & $\mathrm{r}_{\mathrm{ap}}$ & $\mathrm{r}_{\mathrm{e}}$ & $\mathrm{r}_{\mathrm{f}}$ & $\mathrm{ERS}$ \\
\hline PLDC1 & 0,24 & 0,85 & 0,91 & 0,45 & 0,63 & 0,95 \\
PLDC2 & 0,23 & 0,93 & 0,89 & 0,53 & 0,71 & 1,02 \\
PLDC3 & 0,21 & 0,95 & 0,94 & 0,56 & 0,74 & 1,00 \\
PLDC4 & 0,22 & 0,96 & 0,98 & 0,63 & 0,79 & 1,03 \\
PLDC5 & 0,19 & 0,96 & 0,96 & 0,67 & 0,82 & 0,96 \\
PLDC6 & 0,19 & 0,93 & 0,99 & 0,64 & 0,80 & 0,93 \\
PLDC7 & 0,19 & 0,97 & 0,98 & 0,65 & 0,81 & 0,97 \\
PLDC8 & 0,17 & 1,00 & 0,96 & 0,64 & 0,81 & 0,95 \\
PLDC9 & 0,17 & 0,97 & 0,98 & 0,63 & 0,79 & 0,93 \\
PLDC10 & 0,14 & 0,84 & 0,93 & 0,54 & 0,72 & 0,72 \\
PL305* & 0,19 & & & & & \\
\hline
\end{tabular}

\footnotetext{
* Média obtida das 10 análises bivariadas.
}

* Average obtained from 10 bivariates analysis.
O maior valor da estimativa de herdabilidade ocorrido no primeiro controle pode ser explicado pela provável padronização de tratamento nutricional pósparto e pelo ajustamento da produção de leite para o efeito do intervalo parto-primeiro controle.

As estimativas de herdabilidade, em relação à da PL305, foram maiores nas PLDC1 a PLDC4 $\mathrm{e}$ iguais nas PLDC5 a PLDC7, sendo menores nas PLDC8 a PLDC10. Strabel \& Szwaczkowski (1997) encontraram herdabilidade para PLDC muito superior à obtida para PL305, enquanto Swalve (1995) e Firat et al. (1997a) observaram maiores valores de herdabilidade para PL305.

Foram observadas na literatura consideráveis desigualdades para as estimativas de herdabilidade, devido às disparidades entre as populações estudadas e aos diferentes métodos de análise utilizados.

A tendência dos valores de herdabilidade, citados pela literatura, envolvendo animais das raças européias, mostram maiores valores na segunda fase da lactação (Wilmink, 1987; Pander et al., 1992; Firat et al., 1997b; Machado, 1997; Tijani et al., 1999), onde é alegado haver menor variação nas produções destes controles, 
devido à influência de meio ambiente ser mais expressiva no início e no final da lactação, contrariando os resultados deste trabalho. Kettunen et al. (1998) verificaram maior valor da herdabilidade no primeirocontrole, confirmando os achados do presente estudo.

Sikka \& Taneja (1981), em vacas Sahiwal, e Albuquerque (1994), com vacas da raça Gir, verificaram aumento nos coeficientes de herdabilidade da produção parcial e produção parcial acumulada, respectivamente, até o terceiro controle e posterior decréscimo. Já Gadini (1985) notou tendência de diminuição da herdabilidade do primeiro ao quinto controle e posterior aumento até o décimo em rebanho da raça Gir.

Os valores obtidos para a correlação genética entre as PLDC com PL305, apresentados na Tabela 3, foram altos, variando de 0,84 a 1,00 , ou seja, mais de $70 \%$ de proporção da variação genética $\left(\mathrm{r}_{\mathrm{a}}^{2}\right)$ é comum a esses eventos biológicos. Os menores valores estimados foram para PLDC1 e PLDC10, sendo que em PLDC8 o valor foi igual à unidade. As PLDC seriam, então, ótimas indicadoras da PL305 e, seleção praticada nestas características proporcionará progresso na PL305, e vice-versa, pois são afetadas, em grande parte, pelos mesmos genes de ação aditiva.

Este fato, de valor próximo ou igual a 1, pode ser causado pelo limitado número de informações disponíveis neste estudo, pois o aplicativo MTDFREML tende a convergir os valores das estimativas de correlação genética para +1 ou -1 e para um máximo local (Van Vleck, 1992). Um maior conjunto de dados ou inclusão de fatores de variação não considerados no modelo, talvez, seja necessário, para obter estimativas com maior precisão (Silva, 1982). Auran (1976) e Machado (1997) também verificaram estimativas de correlação genética entre PLDC e PL305 igual a unidade.

Os valores estimados para estas correlações genéticas foram semelhantes aos relatados em outros trabalhos (Van Vleck \& Henderson, 1961a; Wilmink,1987; Pander etal., 1992, Gadini, 1997; Ferreira, 1999), sendo os maiores valores encontrados no meio da lactação.

Menores valores estimados de correlação fenotípica foram encontrados também em PLDC1 e PLDC10. As correlações fenotípicas tiveram valores intermediários entre as genéticas e as residuais (Tabela 3).

As correlações residuais foram as mais baixas (Tabela 3), sendo que a correlação entre PLDC1, PLDC2, PLDC3 e PLDC10 com PLDC305 foram inferiores, indicando que os fatores ambientes que afetam as PLDC e PL305 não são os mesmos.

As correlações de ambiente permanente (Tabela 3) tiveram os maiores valores, sugerindo que existem fatores de ambiente comuns que afetam as diferentes lactações.

Utilizando os valores das estimativas de herdabilidade e correlações genéticas para o cálculo da eficiência relativa de seleção (ERS), foi constatado que se a seleção for baseada em PLDC2 a PLDC4 ocorrerá a mesma ou melhor resposta do que quando realizada na PL305 (Tabela 3). Ribas \& Perez (1990) encontraram melhor ERS para o quinto e sexto controles. Com rebanho Gir, Albuquerque (1984) concluiu, com base na ERS, que a seleção para produções de leite acumuladas aos 90 e 120 dias de lactação poderá levar a melhor ganho genético para a produção total.

Assim, com intuito de se obter ganho genético na PL305, pode ser recomendado praticar a seleção indireta baseada nas PLDC2 a PLDC4, cujas avaliações podem, inclusive, ser mais precisas, de acordo com Trus \& Buttazzoni (1990), Pander et al. (1992), Stanton et al. (1992), Van Tassel et al. (1992), Ptak \& Schaeffer (1993), Reents et al. (1995), Swalve (1995), Wiggans \& Goddard (1997) e Ferreira (1999), as quais são mais fáceis de serem obtidas que a produção total de leite, reduzindo custos e o tempo requerido para sua obtenção.

Por outro lado, animais com apenas uma medida de produção podem ser incluídos nas avaliações, o que permite agregar mais informações ao banco de dados e melhorara precisãodasestimativas da PL305, minimizando vícios por descartes de lactações incompletas ou não encerradas (Fimland, 1983; Ptak \& Schaeffer, 1993).

Observando os resultados das análises efetuadas para estimar correlações genéticas entre as PLDC, apresentados na Tabela 4, verifica-se que as estimativas de correlações genéticas entre PLDC1 a PLDC3 com a PLDC10 não foram elevadas, tanto quanto as outras, correspondendo a apenas 41,58 e $55 \%$ de variação comum devido aos genes aditivos $\left(\mathrm{r}_{\mathrm{a}}{ }^{2}\right)$.

Este fato nos induz a questionar qual seria o efeito da seleção, com base na produção de leite nesses controles, sobre a persistência da lactação, apesar de altamente correlacionadas com a PL305. Convém ressaltar, porém, o fato de que a correlação genética entre a PLDC10 e a PL305 foi a menor, com baixa eficiência relativa de seleção (Tabela 3 ).

Os resultados apresentados na Tabela 4 mostram que as correlações genéticas entre as PLDC2 a PLDC8 com a PLDC9 foram altas, indicando que mais de $70 \%$ da variação genética $\left(\mathrm{r}_{\mathrm{a}}{ }^{2}\right)$ nesta pode ser atribuída à influência das outras. As segundas, terceiras e quartas produções apresentaram, além

R. Bras. Zootec., v.31, n.5, p.1953-1963, 2002 
Tabela 4 - Estimativas das correlações genética (ra), residual (re) e fenotípica (rf) entre as produções de leite no dia do controle (PLDC1 a PLDC10)

Table 4 - Genetic (ra), residual (re) and phenotypic (rf) correlations estimated between milk production at the control day (PLDC1 to PLDC10)

\begin{tabular}{|c|c|c|c|c|c|c|}
\hline $\begin{array}{l}\text { Característica } \\
\text { Trait }\end{array}$ & & PLDC6 & PLDC7 & PLDC8 & PLDC9 & PLDC10 \\
\hline \multirow[t]{3}{*}{$\overline{\mathrm{PLDC}} 1$} & ra & 0,82 & 0,83 & 0,82 & 0,71 & 0,64 \\
\hline & re & 0,38 & 0,25 & 0,25 & 0,21 & 0,17 \\
\hline & rf & 0,56 & 0,47 & 0,45 & 0,40 & 0,35 \\
\hline \multirow[t]{3}{*}{ PLDC2 } & ra & 0,95 & 0,92 & 0,93 & 0,83 & 0,76 \\
\hline & re & 0,50 & 0,39 & 0,36 & 0,30 & 0,26 \\
\hline & rf & 0,68 & 0,59 & 0,56 & 0,49 & 0,44 \\
\hline \multirow[t]{3}{*}{ PLDC3 } & ra & 0,96 & 0,98 & 0,99 & 0,88 & 0,74 \\
\hline & re & 0,62 & 0,52 & 0,42 & 0,33 & 0,33 \\
\hline & rf & 0,78 & 0,70 & 0,61 & 0,52 & 0,49 \\
\hline \multirow[t]{3}{*}{ PLDC4 } & ra & 0,99 & 0,96 & 0,99 & 0,90 & 0,81 \\
\hline & re & 0,69 & 0,56 & 0,49 & 0,40 & 0,35 \\
\hline & rf & 0,83 & 0,73 & 0,67 & 0,59 & 0,45 \\
\hline \multirow[t]{3}{*}{ PLDC5 } & ra & 0,98 & 0,99 & 0,99 & 0,94 & 0,89 \\
\hline & re & 0,74 & 0,65 & 0,55 & 0,47 & 0,42 \\
\hline & rf & 0,88 & 0,80 & 0,71 & 0,64 & 0,58 \\
\hline \multirow[t]{3}{*}{ PLDC6 } & ra & & 1,00 & 0,95 & 0,92 & 0,78 \\
\hline & re & & 0,73 & 0,66 & 0,57 & 0,51 \\
\hline & If & & 0,87 & 0,80 & 0,71 & 0,64 \\
\hline \multirow[t]{3}{*}{ PLDC7 } & ra & & & 1,00 & 0,98 & 0,85 \\
\hline & re & & & 0,71 & 0,59 & 0,51 \\
\hline & rf & & & 0,86 & 0,76 & 0,66 \\
\hline \multirow[t]{3}{*}{ PLDC8 } & $\mathrm{ra}$ & & & & 1,00 & 0,83 \\
\hline & re & & & & 0,73 & 0,61 \\
\hline & rf & & & & 0,87 & 0,73 \\
\hline \multirow[t]{3}{*}{ PLDC9 } & ra & & & & & 1,00 \\
\hline & re & & & & & 0,73 \\
\hline & rf & & & & & 0,86 \\
\hline
\end{tabular}

disto, perfeita ERS com a PL305 (Tabela 3).

Outro fato é que a raça Gir submetida à seleção para leite não apresenta problema de persistência de lactação. A duração de lactação média observada para lactações em até 305 dias foi $273,72 \pm 48,95$ dias, sendo que $70,80 \%$ das vacas apresentaram lactações acima de 9 meses (Tabela 5). Martinez et al. (1998) encontraram $81 \%$ das lactações de vacas da raça Gir participantes do teste de progênie com duração igual ou superior a oito meses.

Como a duração da lactação está estreitamente
Tabela 5 - Frequências por classes da duração de lactação

Table 5 - Frequency for lactation length classes

Classes da duração de lactação (dias) $\quad$ Frequência (\%)

Lactation length classes (days)

\begin{tabular}{lr}
\hline $90-120$ & 2,8 \\
$121-150$ & 2,6 \\
$151-180$ & 1,8 \\
$181-210$ & 3,2 \\
$211-240$ & 6,7 \\
$241-270$ & 12,1 \\
$271-300$ & 23,2 \\
$301-305$ & 47,6 \\
\hline
\end{tabular}


associada à produção total de leite, o que pode preocupar é utilizar as PLDC em animais de rebanhos Gir não selecionados para produção de leite, pois a comparação de produções de vacas com diferentes durações de lactações seria inadequado, como preditor do seu potencial produtivo para PL305.

\section{Conclusões}

As correlações genéticas entre as PLDC e PL305 foram altas, bem como as estimativas de herdabilidade de algumas PLDC foram iguais ou mais elevadas que aquelas da PL305, indicando que as PLDC podem ser usadas como critério de seleção, para substituir ou complementar as avaliações baseadas na PL305.

É viável a utilização da PLDC2 a PLDC4 como critério de avaliação dos animais, ocorrendo igual ou melhor eficiência relativa de seleção em relação à PL305, reduzindo o intervalo de gerações.

Embora extremamente sedutora a concepção da avaliação de animais com base nos primeiros controles leiteiros, recomendam-se novas pesquisas para determinar e aquilatar particularidades desse preceito, investigando o número ideal de controles por lactação e/ou múltiplas PLDC agregadas ao modelo de PL305.

\section{Literatura Citada}

ALMANAQUE ABRIL. São Paulo: Editora Abril, 1999. 833p. ANUALPEC 2000: anuário da pecuária brasileira. São Paulo: FNP Consultoria \& Comércio: Ed. Argos, 2000. 392p.

ASBIA. Disponível em: <http//www.asbia.org.br>. Acesso em março de 2002.

ALBUQUERQUE, L.G. Parâmetros genéticos das produções de leite parciais acumuladas de um rebanho Gir no Estado de São Paulo. Ribeirão Preto: Universidade de São Paulo, 1984. 79p. Dissertação (Mestrado em Genética) Universidade de São Paulo, 1984.

AURAN, T. Studies on monthly and cumulative monthly yield records. III. Estimatives of genetic parameters. Acta Agriculture Scandinavia, v.26, n.1, p.3-9, 1976.

BIANCHINI SOBRINHO, E. Estimativa da produção total de leite de vacas da raça Gir, baseada em controles semanais, quinzenais, mensais e bimestrais, obtenção de fatores multiplicativos. Jaboticabal: Universidade Estadual Paulista, 1988. 90p. Tese (Livre Docente) - Universidade Estadual Paulista, 1988.

BIANCHINI SOBRINHO, E. Estudo da curva de lactação de vacas da raça Gir. 1984. 88p. Tese (Doutor em Genética) - Faculdade de Medicina de Ribeirão Preto, Universidade de São Paulo, Ribeirão Preto, 1984.

BOLDMAN, K.G.; KRIESE, L.A.; Van VLECK, L.D. et al. A Manual for use of MTDFREML. A set of programs to obtain estimative of variances and covariances [DRAFT]. Beltsville: Department of Agriculture, Agricultural Research, 1995. 125p.

BRASIL - Ministério da Agricultura, Abastecimento e Reforma
Agrária. Normas técnicas para execução do serviço de controle leiteiro em bovídeos. DOU - Diário Oficial da República Federativa do Brasil, Brasília, DF, 15 out. 1986, n. 195, Seção I, p.15532 a 15535.

CASTLE, O.M.; SEARLE, S.R. Use of bimonthly records in herd testing. Journal of Dairy Science, v. 44, n.7, p.1335-1343, 1961.

CENSO. Disponível em: $<$ http//www.ibge.gov.br/estatística/econo$\mathrm{mia} /$ agropecuária/censoagro/default.shtm $>$. Acessoem: mar 2000.

DANELL, B. Studies on lactation yield and individual test day yields of Swedish dairy cows. II. Estimates of genetic and phenotypic parameters. Acta Agriculture Scandinavia, v.32, n.1, p.83-91, 1982.

FARIA, V.P.; CORSI, M. Índices de produtividade em gado de leite. In: Bovinocultura leiteira - fundamentos da exploração racional. Piracicaba: Fundação de Estudos Agrários "Luiz de Queiroz", 1986, p.1-16.

FERREIRA, W. J. Parâmetros genéticos para produção de leite no dia do controle de vacas da raça Holandesa. Viçosa, MG: Universidade Federal de Viçosa, 1999. 103p. Dissertação (Mestrado emZootecnia) - Universidade Federal de Viçosa, 1999.

FIMLAND, E. Some properties of using the additive genetic relationship in the mixed model technique. Zeitschrift fuer Tierzuchtungund Zuechtungsbiologie, v.100, p.361-374, 1983.

FIRAT, M.Z.; THEOBALD, C.M.; THOMPSON, R. Univariate analysis of test day milk yields of British Holstein-Friesian heifers using Gibbs sampling. Acta Agriculture Scandinavia, v.47, n.4, p.213-220, 1997a.

FIRAT, M.Z.; THEOBALD, C.M.; THOMPSON, R Multivariate analysis of test day milk yields of British Holstein-Friesian heifers using Gibbs sampling. Acta Agriculture Scandinavia, v.47, n.4, p.221-220, 1997b.

GADINI, C.H. Influências genéticas e ambientes sobre a curva de lactação de um rebanho Gir. Ribeirão Preto: Universidade de São Paulo, 1985. 96p. Dissertação (Mestrado em Genética) - Universidade de São Paulo, 1985.

GADINI, C.H. Genetic evaluations of test day production traits and somatic cell scores. Lincoln: University of Nebraska, 1997. 91p. Dissertation (Doctor of Philosophy) - University of Nebraska, 1997.

GOMES, S.T. Transformações na cadeia do leite. Jornal do Produtor de Leite, v.9, n.9, p.1, 1997.

JAMROZIK, J.; KISTEMAKER, G.J.; DEKKERS, J.C.M. et al. Comparison of possible covariates for use in a random regression model for analyses of test day yield. Journal of Dairy Science, v.80, n.10, p.2550-2556, 1997.

KEOWN, J.F.; Van VLECK, L.D. Selection on test day fat percentage and milk production. Journal of Dairy Science, v.54, n.2, p.199-203, 1971.

KETTUNEN, J.F.; MÃNTYSAARI, I.; STRANDÉN, I. et al. Estimation of genetic parameters for first lactation test day milk production using random regression models. In: WORLD CONGRESS ON GENETICS APPLIED TO LIVESTOCK PRODUCTION, 6., 1998, Armidale. Proceedings... Armidale: UNE, 1998, v.23, p.307-310.

LAMB, R.C.; McGILLIARD, L.D. Usefulness of part records to estimate the breeding values of dairy cattle. Journal of Dairy Science, v.50, n.9, p.1459-1467, 1967.

LEDIC,I.L. OGirLeiteiro. DBO Rural, v.14,n.181, p.113-132, 1995.

LEDIC, I.L. Aplicação prática das provas de progênie do Gir Leiteiro a nível de rebanho Gir puro e em vacas mestiças. In: SEMINÁRIO INTERNACIONAL DE MONTERIA DE GANADO DE DOBLE PROPÓSITO, GYR-LECHERO Y

R. Bras. Zootec., v.31, n.5, p.1953-1963, 2002 
BUfALOS, 1., 1996, Monteria. Memorias... Monteria: PRODESA, 1996. p.94-105.

LEDIC, I.L. Gir: o grande trunfo da nossa pecuária leiteira. São Paulo: Peirópolis, 2000. 91p.

LEDIC, I.L.; TONHATI, H. O impacto mercadológico da implantação do programa nacional de melhoramento do Gir Leiteiro. In: SIMPÓSIO NACIONAL DA SOCIEDADE BRASILEIRA DE MELHORAMENTO ANIMAL, 2., 1998, Uberaba. Anais... Uberaba: Sociedade Brasileira de Melhoramento Animal, 1998. p.407-409.

MADDEN, D.E.; McGILLIARD, L.D.; RALSTON, N.P. Relations between test-day milk production of Holstein cows. Journal of Dairy Science, v.42, n.2, p.319-326, 1959.

MACHADO, S.G. Parâmetros genéticos e de ambiente da produção de leite no dia do controle da primeira lactação de vacas da raça Holandesa. Jaboticabal: Universidade Estadual Paulista, 1997. 76p. Dissertação (Mestrado em Zootecnia) - Universidade Estadual Paulista, 1997.

MARTINEZ, M.L.; VERNEQUE, R.S.; TEODORO, R.L. Informativo Gir Leiteiro. Juiz de Fora: CNPGL/EMBRAPA, 1998. 11p.

MEYER, K. Estimating variances and covariances for multivariate animal models by restricted maximum likelihood. Genetique Selection Evolution, v.23, n.1, p.67-83, 1991.

MICROSOFT. Microsoft ${ }^{\circledR}$ Corporation Advanced Software, Inc. Santa Rosa, 1997.

PANDER, B. L.; HILL, W. G.; THOMPSON, R. Genetic parameters of test day records of British Holstein-Friesian heifers. Animal Production, v.55, n.1, p.11-21, 1992.

PEREIRA, J.C.C. Melhoramento genético aplicado à produção de leite. Belo Horizonte: FEPMVZ, 1998. 170p.

PTAK, E.; SCHAEFFER, L.R. Use of test day yields for genetic evaluation of dairy sires and cows. Livestock Production Science, v.34, n.1-2, p.23-34, 1993.

RAO, M.K.; SUNDARESAN, D. Influence of environmental and heredity on the shape of lactation curves in Sahiwal cows. Journal of Agriculture Science, v.92, p.393-401, 1979.

REENTS, R.; DEKKERS, J.C.M.; SCHAEFFER, L.R. Genetic evaluation for somatic cell score with a test day model for multiple lactations. Journal of Dairy Science, v.78, n.12, p.2858-2870, 1995.

REKAYA, R.; BÉJAR, F.; CARABAÑO, M.J. et al. Genetic parameters for test day measurements in spanish HolsteinFriesian. In: INTERBULL ANNUAL MEETING, 10., 1995, Prague. Proceeding... Prague: 1995. n.11, p.1-8.

RIBAS, M.; PEREZ, B. Monthly test day milk records and yield at 244 days. II. Genetic parameters in first lactation. Cuban Journal of Agriculture Science, v.24, n.2, p.139-144, 1990.

SAMPAIO, I.B.M. Estatística aplicada à experimentação animal. Belo Horizonte: FEPMVZ, 1998. 221p.

SAS INSTITUTE SAS/STAT ${ }^{\circledR}$ user's guide. version 6. 4.ed. Cary: 1990. 1290p.

SCHUTZ, M.M.; HANSEN, L.B.; STEUERNAGEL, G.R. Variation of milk, fat, protein and somatic cells for dairy cattle. Journal of Dairy Science, v.73, n.2, p.484-493, 1990.

SEARLE, S.R. Part lactations. II. Genetic phenotypic studies of monthy milk fat yield. Journal of Dairy Science, v.44, n.2, p. 282-294, 1961.

SILVA, R.G. Métodos de genética quantitativa aplicados ao melhoramento animal. Ribeirão Preto: Sociedade Brasileira de Genética, 1982. 162p.

SIKKA, A.K.; TANEJA, V.K. Inheritage of part lactation records in Sahiwal cattle. Indian Journal of Animal Science, v. 51, n.9, p.809-811, 1981.

STANTON, T.L.; JONES, L.R.; EVERETT, R.W. et al. Estimating milk, fat and protein lactation curves with a test day model.
Journal of Dairy Science, v.75, n.6, p.1691-1700, 1992. STRABEL, T.; SZWACZKOWSKI, T. Additive genetic and permanent environmental variance components for test day milk traits in Black-White cattle. Livestock Production Science, v.48, n.2, p.91-98, 1997.

SWALVE, H.H. Genetic relationships between test day milk production and persistency in dairy cattle performance. In: THE WORLD CONGRESS ON GENETICS APPLIED TO LIVESTOCK PRODUCTION, 5., 1994, Guelph. Proceedings... Guelph, 1994. v.18, p.467-470.

SWALVE, H.H. The effect of test day models on the estimation of genetic parameters and breeding values for dairy yield traits. Journal of Dairy Science, v.78, n.4, p.929-938, 1995.

TIJANI, A.; WIGGANS, G.R.; Van TASSEL, C.P. et al. Use of (co)variance function to describe (co)variances for test day yield. Journal of Dairy Science, v.82, n.1, p.226, 1999.

TRUS, D.; BUTTAZZONI, L.G. A multiple trait approach to modelling the lactation curve. In: WORLD CONGRESS ON GENETICS APPLIED TO LIVESTOCK PRODUCTION, 4., 1990, Edinburgh. Proceedings... Edinburgh: UK, 1990. v.13, p.492-495.

Van TASSEL, C.P.; QUASS, R.L.; EVERETT, R.W. Parameter estimates for 305-day ME records and 305-day test day residual records. Journal of Dairy Science, v.75, p.251, 1992. (suppl. 1)

Van VLECK, L.D. The resolution in statistical computing: from least squares to DFREML. In: NATIONAL BREEDERS ROUNDTABLE, POULTRY BREEDERS OF AMERICA AND SOUTH EASTERN POULTRY AND EGG. ASSOCIATION, 41., 1992, St. Louis. Proceedings... St. Louis: 1992. p.1-32.

Van VLECK, L.D.; HENDERSON, C.R. Regression factors for predicting a succeeding complete lactation milk record from part lactation records. Journal of Dairy Science, v.44, n.6, p.1322, 1961a.

Van VLECK, L.D.; HENDERSON, C.R. Use of part lactation records in sire evaluation. Journal of Dairy Science, v.44, n. 8, p.511-1518, 1961b.

VARGAS, B.; PEREZ, E.; Van ARENDONK, J.A.M. Analysis of test day yield data of Costa Rican dairy cattle. Journal of Dairy Science, v.81, n.1, p.255-263, 1998.

VERNEQUE, R.S. Procedimentos numéricos e estimação de componentes de covariância em análise multivariada pelo método da máxima verossimilhança restrita: modelos mistos aplicados ao melhoramento animal. Piracicaba: Escola Superior de Agricultura Luiz de Queiroz, 1994. 155p. Tese (Doutorado em Estatística e Experimentação Animal) Escola Superior de Agricultura Luiz de Queiroz, 1994.

VERNEQUE, R.S.; MARTINEZ, M.L.; TEODORO, R.L et al. Programa nacional de melhoramento do Gir Leiteiro. Resultados do teste de progênie $-\mathbf{1 0}^{\circ}$ grupo. Juiz de Fora: Embrapa Gado de Leite, 2002. 32p. (Documentos, 84).

VIANA, J.A.C. O terceiro mundo não é assim: está assim. Belo Horizonte: FEP/MVZ, 1999. 689p.

WIGGANS, G.R.; GODDARD, M.E. A computationally feasible test day model for genetic evaluation of yield traits in the United States. Journal of Dairy Science, v.80, n.8, p.1795-1800, 1997.

WILMINK, J.B.M. Efficiency of selection for different cumulative milk, fat and protein yields in first lactation. Livestock Production Science, v.17, n.3, p.211-224, 1987.

Recebido em: 06/03/02 Aceito em: 12/06/02

R. Bras. Zootec., v.31, n.5, p.1953-1963, 2002 